VOL. 7 (1972), 121-130.

\title{
Asymptotic expansion of integrals occurring in linear wave theory
}

\section{P. van den Driessche and R.D. Braddock}

The asymptotic expansion of an integral of the type $\int_{-\infty}^{\infty} \Psi(k) \exp (i t \phi(k)) d k$, is derived in terms of the large parameter $t$. Functions $\phi(k)$. and $\Psi(k)$ are assumed analytic, and $\Psi(k)$ may have zeros at a stationary phase point. The usual one dimensional stationary phase and Airy integral terms are found as special cases of a more general result. The result is used to find the leading term of the asymptotic expansion of the double integral. A particular two dimensional $\phi(k)$ relevant to surface water wave problems is considered in detail, and the order of magnitude of the integral is shown to depend on the nature of $\Psi(k)$ at the stationary phase point.

\section{Introduction}

A type of integral frequently encountered in the solution of linear wave problems is

$$
L(t)=\int_{-\infty}^{\infty} \Psi(k) \exp (i t \phi(k)) d k,
$$

where $k$ is the wave number, $\phi(k)$ is the phase function and $\Psi(k)$ is the amplitude function. Interest in applications often centres on the asymptotic value of $L$ for large values of the time, $t$.

When $k$ is confined to one dimension the usual method of stationary

Received 16 March 1972. 
phase can be used, see for example Copson [3]; this must be modified at points where the phase function has a double stationary point (Jeffreys and Jeffreys [7]). The method of stationary phase can be extended to higher dimensions, and must be combined with the method of residues when the amplitude function has singularities (Lighthill [11, 12]; Jones [8]). It also breaks down when the amplitude function is zero at a stationary phase point.

Double integrals similar to (1) but with a finite domain of integration have been considered in detail by Jones and Kline [9], Chako [2] and De Kok [4]; explicit asymptotic expansions of the integral are derived by evaluating contributions from various interior and boundary stationary points of the phase function.

This paper deals with one and two dimensional integrals of type (1) and determines the modifications necessary when the amplitude function vanishes at a stationary phase point. The investigation was prompted by a model for tsunami generation forced by an asymmetric bottom velocity. The amplitude function is then zero at the long wave length limit, which is a physically important region.

\section{One dimensional integral}

Consider the one dimensional integral (1) with $\phi(k)$ and $\Psi(k)$ analytic functions of $k$. Assuming that $k=k^{*}$ is the only stationary phase point, the major contribution to $L$ for large values of $t$ is obtained from the neighbourhood of $k=k^{*}$. Setting $u=k-k^{*}$,

$$
L \approx \int_{-\varepsilon}^{\varepsilon} \Psi(u) e^{i t \phi(u)} d u
$$

where $\varepsilon$ is small and positive. Now

$$
\phi(u)=\phi_{0}+\sum_{s=q}^{\infty} \phi_{s} u^{s} / s !, \Psi(u)=\sum_{p=r}^{\infty} \Psi_{p} u^{p} / p !,
$$

where $r, q$ are integers, $r \geq 0, q \geq 2$, and $\phi_{s}$ is understood to be evaluated at the stationary phase point, that is, 


$$
\phi_{s}=\left(\frac{d^{s} \phi(u)}{d u^{s}}\right)_{u=0}
$$

Note that zero terms have been omitted from the expressions in (2); if the stationary phase point is of order $q-1$, then $\phi_{1}, \phi_{2}, \ldots, \phi_{q-1}$ are all zero. Approximating $\phi$ by the first two non-zero terms yields

$$
L \& \sum_{p=r}^{\infty} \Psi_{p} \int_{-\varepsilon}^{\varepsilon} F^{p, q}(u) d u
$$

where $F^{p, q}(u)=\exp \left(i t\left(\phi_{0}+\phi_{q} u^{q} / q !\right)\right) u^{p} / p !$.

If $q$ is even, $p^{p, q}(u)$ is odd or even according as $p$ is odd or even, and so

$$
L \approx 2 \sum_{p=m}^{\infty} \Psi_{2 p} \int_{0}^{\infty} F^{2 p, q}(u) d u
$$

where $m=[(p+1) / 2]$. The odd terms give zero on integration, and the range of integration for the even terms can be expanded since contributions from $(\varepsilon, \infty)$ are negligible.

$$
\text { If } q \text { is odd, }
$$

(4) $L \approx \sum_{p=s}^{\infty} \exp \left(i t \phi_{0}\right)\left\{(-1)^{p} \int_{0}^{\infty} u^{p} \exp \left(-i t \phi_{q} u^{q} / q !\right) d u\right.$

$$
\left.+\int_{0}^{\infty} u^{p} \exp \left(i t \phi_{q} u^{q} / q !\right) d u\right\} \psi_{p} / p !
$$

The particular cases arising in (3) and (4) lead to a consideration of an integral of the form

$$
J_{ \pm}^{p, q}=\int_{0}^{\infty} u^{p} \exp \left( \pm i t b u^{q} / q !\right) d u
$$

where $b$ is a positive constant. Set $v=u^{q} / q !$, whence

$$
J_{ \pm}^{p, q}=(q !)^{(p+1) / q-1}(q-1) ! \int_{0}^{\infty} v^{(p+1) / q-1} \exp ( \pm i t b v) d v,
$$

which can be evaluated in terms of a gamma function as 
(5) $J_{ \pm}^{p, q}=(q !)^{(p+1) / q-1}(q-1) ! \Gamma((p+1) / q)(b t)^{-(p+1) / q} \exp \left( \pm \frac{1}{2}(p+1) \pi i / q\right)$.

The result (5) can now be used to write down asymptotic approximations to the integrals. If $q$ is even (3) gives

(6) $L \approx 2(q-1) ! \exp \left(i t \phi_{0}\right) \sum_{p=m}^{\infty} \psi_{2 p} \frac{(q !)^{(2 p+1) / q-1}}{(2 p) !} \Gamma((2 p+1) / q)$

$$
\left(\left|\phi_{q}\right| t\right)^{-(2 p+1) / q} \exp \left(\frac{1}{2}(2 p+1) \pi i / q \operatorname{sgn} \phi_{q}\right) \text {. }
$$

Notice that if $q$ is even the asymptotic approximations for $r$ odd or even are of the same order. If $q$ is odd (4) gives

$$
\text { (7) } \begin{aligned}
L \approx 2(q-1) ! \exp \left(i t \phi_{0}\right) & \sum_{p=r}^{\infty} \psi_{p} \frac{(q !)^{(p+1) / q-1}}{p !} \Gamma((p+1) / q) \\
& \left(\left|\phi_{q}\right| t\right)^{-(p+1) / q} \exp \left(\frac{1}{2} i \pi p\right) \cos \left(\frac{1}{2}(p+1) \pi / q \operatorname{sgn} \phi_{q}-\frac{1}{2} \pi p\right) .
\end{aligned}
$$

For large values of $t$ the first term in the series gives the major contribution to $L$; this arises from the first or second non-zero derivative of $\Psi$ at $k=k^{*}$. When $\phi$ has a finite number of stationary points the contributions from each are additive. Note that the above expressions can be extended to the case in which $r$ and $q$ are not integers but satisfy $x+1>-q$, an inequality needed in establishing (5). In such a case the factorial quantities are replaced by gamma functions.

The order of magnitude of the leading term in some frequently encountered examples are listed in Table 1 on page 125. 


\begin{tabular}{cll}
\hline $\begin{array}{c}\text { Conditions on } \\
\phi \text { and } \Psi\end{array}$ & $\begin{array}{l}\text { Order of } \\
\text { magnitude }\end{array}$ & Reference \\
$r=0, q=2$ & $t^{-\frac{1}{2}}$ & usual stationary phase: Copson [3] \\
$r=1, q=2$ & $t^{-\frac{3}{2}}$ & Jones [8], p. 345 \\
general $r, q=2$ & $t^{-\left(\frac{1}{2}+m\right)}$ & Fang and Klosner [5], equation (48) \\
$r=0, q=3$ & $t^{-\frac{1}{3}}$ & Airy integral. Jeffreys and \\
$r=1, q=3$ & $t^{-\frac{2}{3}}$ & Jeffreys [7] \\
\hline
\end{tabular}

Table 1. The order of magnitude of $\int_{-\infty}^{\infty} \Psi(k) e^{i t \phi(k)} d k$ for large $t$; $\phi$ and $\Psi$ given by $(2), m=[(r+1) / 2]$.

\section{Two dimensional integral*}

Consider the two dimensional integral (1) with $\mathrm{k}=(k, 2)$; and $\phi(k), \Psi(k)$ analytic functions of $k$. It is assumed that $\left(k^{*}, z^{*}\right)$ is the only stationary point of $\phi(k)$. Taking $u=k-k^{*}, v=\imath-l^{*}$, this gives the first partial derivatives zero at the origin, namely $\phi_{10}=\phi_{01}=0$; and it is further assumed that $\phi_{20} \phi_{02}-\phi_{11}^{2} \neq 0$, see (8) below, where

$$
\phi_{p q}=\left(\frac{\partial^{p+q} \phi}{\partial u^{p} \partial v^{q}}\right)_{u=0, v=0}
$$

Initially the amplitude function is taken to be non-zero at the stationary phase point, that is, $\Psi_{00} \neq 0$. Approximating $\phi$ by the terms

$$
\phi=\phi_{00}+\frac{1}{2}\left(\phi_{20} u^{2}+2 \phi_{11} u v+\phi_{02} v^{2}\right) \text {, }
$$

(1) becomes

$$
L \approx \Psi_{00} \exp \left(i t \phi_{00}\right) \int_{-\infty}^{\infty} \int_{-\infty}^{\infty} \exp \left(\frac{1}{2} i t\left(\phi_{20} u^{2}+2 \phi_{1} u v+\phi_{02} v^{2}\right)\right) d u d v .
$$

* This section elaborates an idea of Lighthill [11]. 
In general $\phi_{11} \neq 0$, and it is now necessary to rotate the $u v$ axes through an angle $\arctan \left(2 \phi_{11} /\left(\phi_{20}-\phi_{02}\right)\right)$ to eliminate this term from the exponential. The resulting integrals can then be estimated using the leading term of (6), giving

$$
L \text { \& } 2 \pi \Psi \Psi_{00}\left(\left|\phi_{20} \phi_{02}-\phi_{11}^{2}\right|\right)^{-\frac{1}{2}} t^{-1} \exp i\left(t \phi_{00}+\frac{1}{4} \pi \sigma\right),
$$

where $\sigma=$ signature $\left(\begin{array}{ll}\phi_{11} & \phi_{12} \\ \phi_{12} & \phi_{22}\end{array}\right)$.

If the amplitude function is zero at the stationary point it can be expanded in a series about the point and the above argument extended as in the case of the one dimensional integral. This is developed by Jones and Kline [9] and by Chako [2], who also deal with the modifications necessary when $\phi_{20} \phi_{02}-\phi_{11}^{2}=0$. In addition contributions from other stationary phase points are additive.

\section{Polar transformation}

In some two dimensional cases it is more convenient to transform (1) into polar coordinates; this is the case for problems dealing with a fluid of depth $h$ with a free upper surface when the phase function is

$$
\phi(k, z)=x k / t+y z / t \pm(g|k| \tanh (|k| h))^{\frac{1}{2}},
$$

which is analytic in $E^{2}-\{(0,0)\}$. Taking the negative sign in the exponential and setting $x=r \cos \theta, y=r \sin \theta, k=|k| \cos n$, $I=|k| \operatorname{sinn}, X=|k| h, \mu(X)=h^{-\frac{1}{2}}(g x \tanh \chi)^{\frac{1}{2}}$, the integral (1) becomes

$$
L=\int_{0}^{\infty} \int_{0}^{2 \pi} \chi^{\Psi}(x, n) \exp (i t \phi(x, n)) d n d x
$$

where

$$
\phi(\chi, n)=(r x \mid(t h)) \cos (\theta-n)-\mu(x) .
$$

Here the range of integration is the phase plane described by the polar coordinates $(x, n)$.

Now suppose there is a stationary phase point at $\left(x^{*}, n^{*}\right)$ with $X^{*} \neq 0$ such that $\phi_{10}=\phi_{01}=0$; suffixes now refer to derivatives with respect to the phase plane variables evaluated at $\left(x^{*}, n^{*}\right)$. In addition 
the above form of $\phi$ implies that $\phi_{11}=0$. Assuming that $\Psi_{00}, \phi_{20}$ and $\phi_{02}$ are non-zero, (8) gives

$$
L \approx 2 \pi x * \Psi_{00}\left(\left.\right|_{20} \phi_{02} \mid\right)^{-\frac{1}{2} t} t^{-1} \exp i\left(t \phi_{00}+\frac{1}{4} \pi\left(\operatorname{sgn} \phi_{20}+\operatorname{sgn} \phi_{02}\right)\right) \text {. }
$$

Assume now that $\Psi$ has a zero at $\left(X^{*}, n^{*}\right)$, but at least one of $\Psi_{20}, \Psi_{02}$ are non-zero; the conditions on $\phi$ are unchanged. The above argument, retaining terms in $\phi$ up to and including second derivatives, yields

$$
\begin{aligned}
& L \approx \pi x^{*}\left(\left|\phi_{20} \phi_{02}\right|\right)^{-\frac{1}{2}} t^{-2}\left\{\Psi_{20}\left(\left|\phi_{20}\right|\right)^{-1} \exp \left(\frac{1}{2} \pi i \operatorname{sgn} \phi_{20}\right)\right. \\
& \left.+\Psi_{02}\left(\left|\phi_{02}\right|\right)^{-1} \exp \left(\frac{1}{2} \pi i \operatorname{sgn} \phi_{02}\right)\right\} \exp i\left(t \phi_{00}+\frac{1}{4} \pi\left(\operatorname{sgn} \phi_{20}+\operatorname{sgn} \phi_{02}\right)\right) .
\end{aligned}
$$

The terms involving $\Psi_{10}$ and $\Psi_{01}$ give odd functions and so zero contribution to the asymptotic approximation. Notice that there are two terms in the approximation (13) both of order $t^{-2}$, in contrast with the result of order $t^{-1}$ from (12) when $\Psi_{00} \neq 0$. If both $\Psi_{20}$ and $\Psi_{02}$ are zero the result (13) needs further modification by considering more terms in the series for $\Psi$. If $n^{*}=0$, the lower limit of $n$, the results of (12) and (13) must be multiplied by $\frac{1}{2}$.

Consider now the phase function $\phi$ given by (11). When $x^{*} \neq 0$ and $r<t(g h)^{\frac{1}{2}}$ there is one stationary phase point $\left(X^{*}, n^{*}\right)$ satisfying $n^{*}=\theta$ and $\mu_{1}=r /(t h)$. The second solution to $\phi_{01}=0$, namely $n=\theta+\pi$, is rejected as it does not satisfy $\phi_{10}=0$. This is due to the nature of $d \mu / d x$, which is a positive, strictly monotonic decreasing function on $(0, \infty)$ with a maximum value of $(g / h)^{\frac{1}{2}}$ at $x=0$. At the stationary phase point $\phi_{20} \phi_{02}=X^{\star} \mu_{1} \mu_{2}$, thus the denominator in (12) (or (13)) does not vanish for $X^{*} \neq 0$. The asymptotic approximations are thus correct except near the long wave length limit. Formula (8) is also invalid here for $\phi$ given by $(9)$ as the denominator terms are undefined at the origin.

\section{Long wave length limit}

When $X^{*}$ is a small positive number, that is at the long wave length 
limit, the approximations obtained previously are invalid. In this case approximations can be achieved by first applying the stationary phase principle to the $\eta$ integral in (10), yielding

$$
L \approx(2 \pi h / r)^{\frac{1}{2}} \int_{0}^{\infty} x^{\frac{1}{2}} \Psi\left(x, n^{*}\right) \exp i\left((r x /(t h)-\mu(x)) t-\frac{1}{4} \pi\right) d x
$$

The resulting integral is similar to the one considered by Weston [13] for the initial part of the received pressure pulse produced by a large explosion in the atmosphere. Weston expresses the integral in terms of products of Airy integrals and is thus able to find an asymptotic form. Here Watson's Lemma is used to yield the leading term of the asymptotic expansion for (14).

The phase in (14) near the stationary point $\chi^{*}$ is approximately $-\frac{1}{4} \pi+t(g / h)^{\frac{1}{2}} \chi^{3} / 3$ ! and the amplitude function is expanded in a series as

$$
\Psi=\sum_{p=q}^{\infty} \Psi_{p} x^{p / p !}, \quad \Psi_{p}=\left(\frac{d^{p} \Psi}{d x^{p}}\right)_{\chi=\chi^{*} \approx_{0}, n=n^{*}} .
$$

At this long wave length $r=\sqrt{g h t}$ from the stationary phase condition, thus

$$
\begin{aligned}
& \text { (15) } L \text { fo }(8 \pi i h / g)^{\frac{1}{2}} \exp \left(-\frac{1}{4} \pi i\right) \sum_{p=q}^{\infty} \Psi_{p} / p !(6)^{(2 p-3) / 6} \Gamma((2 p+3) / 6) \\
& \left(g^{\frac{1}{2}} h^{-\frac{1}{2}}\right)^{-p / 3} t^{-(p / 3+1)} \operatorname{exp\pi i}((2 p+3) / 12) .
\end{aligned}
$$

Thus when $\Psi\left(x^{*}, n^{*}\right) \neq 0$ this has the same order, namely $t^{-1}$, as the main wave, a result which agrees with Gazarian [6] and Kajiura [10]. In agreement with Gazarian the ratio of amplitudes of the main wave to the leading wave is $\sqrt{3}$. However when $\Psi$ does vanish at the long wave length limit the order becomes $t^{-(q / 3+1)}$. Thus when $\Psi$ has a simple zero the order is $t^{-4 / 3}$, and when $\Psi$ has a double zero the order is $t^{-5 / 3}$. In these cases the main wave (order $t^{-1}$ ) has a larger magnitude than the leading wave. 


\section{References}

[1] R.D. Braddock and P. van den Driessche, "Tsunami generation", Proc. Fifteenth General Assembly, IUGG, Moscow, 1971 (to be published).

[2] Nicholas Chako, "Asymptotic expansions of double and multiple integrals occurring in diffraction theory", J. Inst. Math. Applic8. (1965), 372-422.

[3] E.T. Copson, Asymptotic expansions (Cambridge Tracts in Mathematics and Mathematical Physics, No. 55. Cambridge University Press, Cambridge, 1965).

[4] F. de Kok, "On the method of stationary phase for multiple integrals", SIAM J. Math. Anaz. 2 (1971), 76-104.

[5] T.C. Fang and J.M. Klosner, "Expanding axial wave on a submerged cylindrical shell", Quart. Appl. Math. 28 (1970), 355-376.

[6] Iu.L. Gazarian, "On surface waves launched in the ocean by underwater earthquakes", Akust. Ž. 1 (1955), 203-217.

[7] Harold Jeffreys and Bertha Swirles Jeffreys, Methods of mathematical physics, lst ed. (Cambridge University Press, Cambridge, 1946).

[8] D.S. Jones, Generalised functions (McGraw-Hill, New York; Toronto; Ontario; London; 1966).

[9] Douglas S. Jones and Morris Kline, "Asymptotic expansion of multiple integrals and the method of stationary phase", J. Math. Phys. 37 (1958), 1-28.

[10] Kinjiro Kajiura, "The leading wave of a tsunami", Buzz. Earthquake Res. Inst. Univ. Tokyo 41 (1963), 535-571.

[11] M.J. Lighthill, "Studies on magneto-hydrodynamic waves and other anisotropic wave motions", Philos. Trans. Roy. Soc. London Ser. A 252 (1960), 397-430.

[12] M.J. Lighthill, "Group velocity", J. Inst. Maths. Applics. 1 (1965), $1-28$. 
[13] V.H. Weston, "The pressure pulse produced by a large explosion in the atmosphere", Canad. J. Phys. 39 (1961), 993-1009.

\author{
Department of Mathematics, \\ University of Victoria, \\ victoria, \\ British Columbia, \\ Canada, \\ and \\ Department of Mathematics, \\ University of Queensland, \\ St Lucia, \\ Queensland.
}

